REPORTS ON MATHEMATICAL LOGIC

52 (2017), 69-82

doi:10.4467/20842589RM.17.004.7142

George VOUTSADAKIS

\title{
CATEGORICAL ABSTRACT ALGEBRAIC LOGIC: WÓJCICKI'S CONJECTURE AND MALINOWSKI'S THEOREM
}

\begin{abstract}
A b s t r a c t. During the Autumn School on Strongly Finite Sentential Calculi held in Miȩdzygórze in 1977, Wójcicki conjectured that a propositional logic has a strongly adequate matrix semantics consisting of matrices with a singleton designated filter, which we call a Rasiowa semantics since it is possessed by all implicative logics of Rasiowa, if and only if it satisfies a simple technical condition that we name the Wójcicki condition. Malinowski proved the conjecture in 1978. We revisit Malinowski's Theorem in the setting of logics formalized as $\pi$-institutions.
\end{abstract}

Received 21 December 2016

Keywords and phrases: sentential logics, logical matrices, implicative logics, Suszko congruence, $\pi$-Institutions, Wójcicki's conjecture, Malinowski's theorem.

AMS subject classification: 03G27. 


\section{Introduction}

Let $\mathcal{L}=\langle\Lambda, \rho\rangle$ be a logical signature/algebraic type, i.e., a set of logical connectives/operation symbols $\Lambda$ with attached finite arities given by the function $\rho: \Lambda \rightarrow \omega$. Let, also, $V$ be a countably infinite set of propositional variables. We denote by $\operatorname{Fm}_{\mathcal{L}}(V)$ the set of $\mathcal{L}$-formulas $/ \mathcal{L}$-terms with variables in $V$ and by $\mathbf{F m}_{\mathcal{L}}(V)$ the corresponding free algebra. A (sentential or propositional) logic $\mathcal{S}=\langle\mathcal{L}, C\rangle$ is a pair, where $\mathcal{L}$ is a logical signature and $C: \mathcal{P}\left(\operatorname{Fm}_{\mathcal{L}}(V)\right) \rightarrow \mathcal{P}\left(\operatorname{Fm}_{\mathcal{L}}(V)\right)$ is a structural closure operator on $\operatorname{Fm}_{\mathcal{L}}(V)$, i.e., a closure operator satisfying, for every endomorphism $h: \mathbf{F m}_{\mathcal{L}}(V) \rightarrow \mathbf{F m}_{\mathcal{L}}(V)$, the structurality condition

$$
h(C(X)) \subseteq C(h(X)) \text {, for all } X \subseteq \operatorname{Fm}_{\mathcal{L}}(V) .
$$

A theory of a logic $\mathcal{S}=\langle\mathcal{L}, C\rangle$ is a $C$-closed set of formulas, i.e., a set $T \subseteq \operatorname{Fm}_{\mathcal{L}}(V)$, such that $C(T)=T$. The set of all theories of $\mathcal{S}$ is denoted by $\operatorname{Th}(\mathcal{S})$.

Given a propositional logic $\mathcal{S}=\langle\mathcal{L}, C\rangle$, the Leibniz congruence $\Omega(T)$ of a theory $T$ of $\mathcal{S}$ (see [1]) is the largest congruence $\theta$ on $\mathbf{F m}_{\mathcal{L}}(V)$ that is compatible with $T$, i.e., such that

$$
\langle\alpha, \beta\rangle \in \theta \quad \text { and } \quad \alpha \in T \text { imply } \beta \in T \text {, }
$$

for all $\alpha, \beta \in \operatorname{Fm}_{\mathcal{L}}(V)$. Blok and Pigozzi revisited in [1] a characterization of the Leibniz congruence, first given by Czelakowski in [2]. It asserts that for all $\alpha, \beta \in \operatorname{Fm}_{\mathcal{L}}(V)$,

$$
\begin{aligned}
\langle\alpha, \beta\rangle \in \Omega(T) \quad \text { iff } & \text { for all } \varphi(p, \vec{q}) \in \operatorname{Fm}_{\mathcal{L}}(V), \\
& \varphi(\alpha, \vec{q}) \in T \text { iff } \varphi(\beta, \vec{q}) \in T .
\end{aligned}
$$

The Tarski congruence $\widetilde{\Omega}(\mathcal{S})$ of $\mathcal{S}$ (see [4]) is the largest congruence relation $\theta$ on $\mathbf{F m}_{\mathcal{L}}(V)$ that is compatible with all theories of $\mathcal{S}$. The Tarski congruence is a special case of the Suszko congruence $\widetilde{\Omega}^{\mathcal{S}}(T)$ associated with a given theory $T$ of $\mathcal{S}$, which is defined as the largest congruence on $\mathbf{F m}_{\mathcal{L}}(V)$ that is compatible with all theories of $\mathcal{S}$ that contain the given theory $T$. (This was defined originally in unpublished notes by Suszko in 1977. See, also, [3].) In fact, by definition, $\widetilde{\Omega}(\mathcal{S})=\widetilde{\Omega}^{\mathcal{S}}(C(\varnothing))$, i.e., the Tarski congruence of $\mathcal{S}$ is the Suszko congruence associated with the set of theorems $C(\varnothing)$ of the logic $\mathcal{S}$. Font and Jansana [4], extending the 
characterization of the Leibniz congruence $\Omega(T)$ associated with a theory $T$ of a sentential logic, have shown that, for all $\alpha, \beta \in \operatorname{Fm}_{\mathcal{L}}(V)$,

$$
\begin{aligned}
&\langle\alpha, \beta\rangle \in \widetilde{\Omega}(\mathcal{S}) \quad \text { iff } \quad \text { for all } \varphi(p, \vec{q}) \in \operatorname{Fm}_{\mathcal{L}}(V), \\
& C(\varphi(\alpha, \vec{q}))=C(\varphi(\beta, \vec{q})) .
\end{aligned}
$$

Further generalizing this characterization, Czelakowski, using the original expression of Suszko, showed in Theorem 1.3 of [3] that, for all $T \in \operatorname{Th}(\mathcal{S})$, $\alpha, \beta \in \operatorname{Fm}_{\mathcal{L}}(V)$,

$$
\begin{aligned}
\langle\alpha, \beta\rangle \in \widetilde{\Omega}^{\mathcal{S}}(T) \quad \text { iff } \quad & \text { for all } \varphi(p, \vec{q}) \in \operatorname{Fm}_{\mathcal{L}}(V), \\
& C(T, \varphi(\alpha, \vec{q}))=C(T, \varphi(\beta, \vec{q})) .
\end{aligned}
$$

Recall that, given a logical signature $\mathcal{L}$, an $\mathcal{L}$-matrix is a pair $\mathfrak{A}=$ $\langle\boldsymbol{A}, F\rangle$, where $\boldsymbol{A}=\left\langle A, \mathcal{L}^{\boldsymbol{A}}\right\rangle$ is an $\mathcal{L}$-algebra and $F \subseteq A$ is a subset of its universe. Given a propositional logic $\mathcal{S}=\langle\mathcal{L}, C\rangle$, an $\mathcal{L}$-matrix $\mathfrak{A}=\langle\boldsymbol{A}, F\rangle$ is called an $\mathcal{S}$-matrix if $F$ is an $\mathcal{S}$-filter, i.e., if, for all $X \cup\{\alpha\} \subseteq \operatorname{Fm}_{\mathcal{L}}(V)$, such that $\alpha \in C(X)$, and all $h: \mathbf{F m}_{\mathcal{L}}(V) \rightarrow \boldsymbol{A}$,

$$
h(X) \subseteq F \quad \text { implies } \quad h(\alpha) \in F .
$$

The class of all $\mathcal{S}$-matrices is denoted by $\operatorname{Mat}(\mathcal{S})$. Each class of $\mathcal{L}$-matrices $\mathrm{M}$ defines a sentential logic $\mathcal{S}^{\mathrm{M}}=\left\langle\mathcal{L}, C^{\mathrm{M}}\right\rangle$ as follows:

$$
\begin{aligned}
\alpha \in C^{\mathrm{M}}(X) \quad \text { iff } & h(X) \subseteq F \Rightarrow h(\alpha) \in F \\
& \text { for all } \mathfrak{A}=\langle\boldsymbol{A}, F\rangle \in \mathrm{M} \text { and all } h: \mathbf{F m}_{\mathcal{L}}(V) \rightarrow \boldsymbol{A} .
\end{aligned}
$$

We write $C^{\mathfrak{A}}$ instead of $C^{\{\mathfrak{A}\}}$. Note that, according to these definitions, $\mathfrak{A}$ is an $\mathcal{S}$-matrix iff $C \leq C^{\mathfrak{A}}$.

In [7], Wójcicki showed that every propositional logic $\mathcal{S}=\langle\mathcal{L}, C\rangle$ is complete with respect to the class of all $\mathcal{S}$-matrices, i.e., that, for all $X \cup$ $\{\alpha\} \subseteq \operatorname{Fm}_{\mathcal{L}}(V)$,

$$
\alpha \in C(X) \quad \text { iff } \quad \alpha \in C^{\operatorname{Mat}(\mathcal{S})}(X) .
$$

This implies that a propositional logic $\mathcal{S}$ is uniquely determined by the class of all $\mathcal{S}$-matrices:

$$
\mathcal{S}_{1}:=\left\langle\mathcal{L}, C_{1}\right\rangle=\left\langle\mathcal{L}, C_{2}\right\rangle=: \mathcal{S}_{2} \quad \text { iff } \quad \operatorname{Mat}\left(\mathcal{S}_{1}\right)=\operatorname{Mat}\left(\mathcal{S}_{2}\right) .
$$

On the other hand, Wójcicki also observed (see [5]) that the implicative logics of Rasiowa [6] satisfy the same property with respect to the smaller class 
of all $\mathcal{S}$-matrices with a singleton $\mathcal{S}$-filter. We call such $\mathcal{S}$-matrices Rasiowa $\mathcal{S}$-matrices and denote their class by $\operatorname{Mat}^{R}(\mathcal{S})$. Rasiowa $\mathcal{S}$-matrices are referred to as $C$-algebras in [5]. All implicative logics of Rasiowa have the completeness property with respect to the class of all Rasiowa $\mathcal{S}$-matrices and, therefore, satisfy

$$
\mathcal{S}_{1}:=\left\langle\mathcal{L}, C_{1}\right\rangle=\left\langle\mathcal{L}, C_{2}\right\rangle=: \mathcal{S}_{2} \quad \text { iff } \quad \operatorname{Mat}^{R}\left(\mathcal{S}_{1}\right)=\operatorname{Mat}^{R}\left(\mathcal{S}_{2}\right) .
$$

We call the determinacy of a logic by the class $\operatorname{Mat}^{R}(\mathcal{S})$ of all its Rasiowa matrix models the Rasiowa semantics property. Dziobiak showed, via an example (Page 21 of [5]), that not all sentential logics satisfy the Rasiowa semantics property.

According to Malinowski [5], during the Autumn School on Strongly Finite Sentential Calculi held in Międzygórze in 1977, Wójcicki conjectured that a sentential logic $\mathcal{S}=\langle\mathcal{L}, C\rangle$ has the Rasiowa semantics property iff, for all $X \cup\{\alpha\} \subseteq \operatorname{Fm}_{\mathcal{L}}(V)$, such that $\alpha \in C(X)$,

$$
C(X)=\left\{\beta \in \operatorname{Fm}_{\mathcal{L}}(V):\langle\beta, \alpha\rangle \in \widetilde{\Omega}^{\mathcal{S}}(C(X))\right\} .
$$

We call this the Wójcicki condition. In the main result of [5] (the Theorem of [5]), Malinowski gives a proof of Wójcicki's conjecture: Using the terminology adopted here, Malinowski proves that

a sentential logic has the Rasiowa semantics property if and only if it satisfies the Wójcicki condition.

In this note, we explore an analog of the Wójcicki-Malinowski Theorem in the context of logics formalized as $\pi$-institutions.

\section{2. $\pi$-Institutions and Closure Systems}

Let Sign $^{b}$ be a category and $\mathrm{SEN}^{b}:$ Sign $^{b} \rightarrow$ Set a Set-valued functor. The clone of all natural transformations on $\mathrm{SEN}^{b}$ is the category $U^{b}$ with collection of objects $\mathrm{SEN}^{\mathrm{b}^{\alpha}}, \alpha$ an ordinal, and collection of morphisms $\tau$ : $\mathrm{SEN}^{b^{\alpha}} \rightarrow \mathrm{SEN}^{b^{\beta}} \beta$-sequences of natural transformations $\tau_{i}: \mathrm{SEN}^{b^{\alpha}} \rightarrow \mathrm{SEN}^{b}$. Composition of $\left\langle\tau_{i}: i<\beta\right\rangle: \operatorname{SEN}^{b^{\alpha}} \rightarrow \operatorname{SEN}^{b^{\beta}}$ with $\left\langle\sigma_{j}: j<\gamma\right\rangle: \operatorname{SEN}^{b^{\beta}} \rightarrow$ SEN $^{b^{\gamma}}$

$$
\mathrm{SEN}^{\mathrm{b}^{\alpha}} \stackrel{\left\langle\tau_{i}: i<\beta\right\rangle}{\longrightarrow} \mathrm{SEN}^{\mathrm{b}^{\beta}} \stackrel{\left\langle\sigma_{j}: j<\gamma\right\rangle}{\longrightarrow} \mathrm{SEN}^{\mathrm{b}^{\gamma}}
$$


is defined by

$$
\left\langle\sigma_{j}: j<\gamma\right\rangle \circ\left\langle\tau_{i}: i<\beta\right\rangle=\left\langle\sigma_{j}\left(\left\langle\tau_{i}: i<\beta\right\rangle\right): j<\gamma\right\rangle .
$$

A subcategory of this category with all objects of the form $\mathrm{SEN}^{\mathrm{b}^{k}}, k<\omega$, and such that:

- it contains all projection morphisms $p^{k, i}: \mathrm{SEN}^{\mathrm{b}^{k}} \rightarrow \mathrm{SEN}^{\mathrm{b}}, i<k, k<\omega$, with $p_{\Sigma}^{k, i}: \operatorname{SEN}^{\mathrm{b}}(\Sigma)^{k} \rightarrow \mathrm{SEN}^{\mathrm{b}}$ given by

$$
p_{\Sigma}^{k, i}(\vec{\phi})=\phi_{i}, \text { for all } \vec{\phi} \in \operatorname{SEN}^{b}(\Sigma)^{k},
$$

- for every family $\left\{\tau_{i}: \mathrm{SEN}^{\mathrm{b}^{k}} \rightarrow \mathrm{SEN}^{\mathrm{b}}: i<\ell\right\}$ of natural transformations in $N^{b},\left\langle\tau_{i}: i<\ell\right\rangle: \mathrm{SEN}^{b^{k}} \rightarrow \mathrm{SEN}^{b^{\ell}}$ is also in $N^{b}$

is referred to as a category of natural transformations on $\mathrm{SEN}^{b}$.

Consider an algebraic system $\boldsymbol{A}^{\mathrm{b}}=\left\langle\mathbf{S i g n}^{\mathrm{b}}, \mathrm{SEN}^{\mathrm{b}}, N^{\mathrm{b}}\right\rangle$, i.e., a triple consisting of

- a category Sign $^{b}$, called the category of signatures;

- a functor $\mathrm{SEN}^{b}: \mathbf{S i g n}^{b} \rightarrow$ Set, called the sentence functor;

- a category of natural transformations $N^{b}$ on SEN ${ }^{b}$.

A $\pi$-institution based on $\boldsymbol{A}^{b}$ is a pair $\mathcal{I}=\left\langle\boldsymbol{A}^{b}, C\right\rangle$, where $C=\left\{C_{\Sigma}\right\}_{\Sigma \in\left|\mathbf{S i g n}^{b}\right|}$ is a closure system on $\mathrm{SEN}^{b}$, i.e., a $\left|\mathbf{S i g n}^{b}\right|$-indexed collection of closure operators $C_{\Sigma}: \mathcal{P S E N}^{\mathrm{b}}(\Sigma) \rightarrow \mathcal{P S E N}^{\mathrm{b}}(\Sigma)$, such that, for all $\Sigma_{1}, \Sigma_{2} \in\left|\mathbf{S i g n}^{\mathrm{b}}\right|$, all $f \in \operatorname{Sign}^{b}\left(\Sigma_{1}, \Sigma_{2}\right)$ and all $\Phi \subseteq \operatorname{SEN}^{b}\left(\Sigma_{1}\right)$,

$$
\operatorname{SEN}^{b}(f)\left(C_{\Sigma_{1}}(\Phi)\right) \subseteq C_{\Sigma_{2}}\left(\operatorname{SEN}^{b}(f)(\Phi)\right) .
$$

This condition is sometimes referred to as structurality by analogy with Condition (1). In this context, $\boldsymbol{A}^{\mathrm{b}}$ is also referred to as the base algebraic system. Given a $\pi$-institution $\mathcal{I}$, a theory family $T^{b}=\left\{T_{\Sigma}^{b}\right\}_{\Sigma \in\left|\mathbf{S i g n}^{b}\right|}$ is a $\left|\mathbf{S i g n}^{b}\right|$-indexed collection of subsets $T_{\Sigma}^{b} \subseteq \operatorname{SEN}^{b}(\Sigma)$, closed under $C_{\Sigma}$, i.e., such that $C_{\Sigma}\left(T_{\Sigma}^{b}\right)=T_{\Sigma}^{b}$, for all $\Sigma \in\left|\mathbf{S i g n}^{\mathrm{b}}\right|$. The collection of all theory families of $\mathcal{I}$ is denoted by $\operatorname{ThFam}(\mathcal{I})$. It is well-known that, ordered by signature-wise inclusion $\leq$, it forms a complete lattice $\operatorname{ThFam}(\mathcal{I})=$ $\langle\operatorname{ThFam}(\mathcal{I}), \leq\rangle$. 
Note, also, that, given a base algebraic system $\boldsymbol{A}^{b}=\left\langle\mathbf{S i g n}^{b}, \mathrm{SEN}^{b}, N^{b}\right\rangle$, the collection of all closure systems based on $\boldsymbol{A}^{b}$ is closed under signaturewise intersections and, hence, forms a complete lattice under the signaturewise ordering, also denoted by $\leq$ :

$$
\begin{aligned}
C^{1} \leq C^{2} \quad \text { iff } & \text { for all } \Sigma \in\left|\mathbf{S i g n}^{b}\right| \text { and all } \Phi \subseteq \operatorname{SEN}^{b}(\Sigma), \\
& C_{\Sigma}^{1}(\Phi) \subseteq C_{\Sigma}^{2}(\Phi) .
\end{aligned}
$$

\section{Rasiowa Matrix Semantics}

Let $\boldsymbol{A}^{b}=\left\langle\operatorname{Sign}^{b}, \mathrm{SEN}^{b}, N^{b}\right\rangle$ be a base algebraic system. Consider an interpreted algebraic system $\mathcal{A}=\langle\boldsymbol{A},\langle F, \alpha\rangle\rangle$, where $\boldsymbol{A}=\langle$ Sign, SEN, $N\rangle$ is an $N^{b}$-algebraic system (see, e.g., Section 2 of [9]) and $\langle F, \alpha\rangle: \boldsymbol{A}^{\mathrm{b}} \rightarrow \boldsymbol{A}$ is an $N^{b}$-morphism. A sentence family of $\mathcal{A}$ is a $|\operatorname{Sign}|$-indexed collection $T=\left\{T_{\Sigma}\right\}_{\Sigma \in|\operatorname{Sign}|}$, such that $T_{\Sigma} \subseteq \operatorname{SEN}(\Sigma)$, for all $\Sigma \in|\operatorname{Sign}|$. The pair $\mathfrak{A}=\langle\mathcal{A}, T\rangle$ is called a matrix system.

Given a matrix system $\mathfrak{A}=\langle\mathcal{A}, T\rangle$, as above, the closure system $C^{\mathfrak{A}}$ on $\boldsymbol{A}^{b}$ is defined, for all $\Sigma \in\left|\mathbf{S i g n}^{b}\right|$ and all $\Phi \cup\{\varphi\} \subseteq \operatorname{SEN}^{b}(\Sigma)$, by $\varphi \in C_{\Sigma}^{\mathfrak{A}}(\Phi)$ if and only if, for all $\Sigma^{\prime} \in\left|\mathbf{S i g n}^{b}\right|$ and all $f \in \operatorname{Sign}^{b}\left(\Sigma, \Sigma^{\prime}\right)$,

$$
\alpha_{\Sigma^{\prime}}\left(\operatorname{SEN}^{b}(f)(\Phi)\right) \subseteq T_{F\left(\Sigma^{\prime}\right)} \quad \text { implies } \quad \alpha_{\Sigma^{\prime}}\left(\operatorname{SEN}^{b}(f)(\varphi)\right) \in T_{F\left(\Sigma^{\prime}\right)} .
$$

Given a $\pi$-institution $\mathcal{I}=\left\langle\boldsymbol{A}^{\mathrm{b}}, C\right\rangle$, the matrix system $\mathfrak{A}=\langle\mathcal{A}, T\rangle$ is called an $\mathcal{I}$-matrix system or a matrix system model of $\mathcal{I}$ in case $C \leq C^{\mathfrak{A}}$, i.e., if, for all $\Sigma \in\left|\mathbf{S i g n}^{b}\right|$ and all $\Phi \cup\{\varphi\} \subseteq \operatorname{SEN}^{b}(\Sigma)$,

$$
\varphi \in C_{\Sigma}(\Phi) \text { implies } \varphi \in C_{\Sigma}^{\mathfrak{A}}(\Phi) .
$$

The collection of all $\mathcal{I}$-matrix systems is denoted by $\operatorname{MatSys}(\mathcal{I})$. For a collection $\mathrm{M}$ of matrix systems, we define

$$
C^{\mathrm{M}}=\bigcap_{\mathfrak{A} \in \mathrm{M}} C^{\mathfrak{A}},
$$

where, on the right, intersection is applied signature-wise.

Let $\mathcal{I}=\left\langle\boldsymbol{A}^{\mathrm{b}}, C\right\rangle$ be a $\pi$-institution. Denote by $\langle I, \iota\rangle: \boldsymbol{A}^{\mathrm{b}} \rightarrow \boldsymbol{A}^{\mathrm{b}}$ the identity morphism and by $\mathcal{A}^{b}=\left\langle\boldsymbol{A}^{b},\langle I, \iota\rangle\right\rangle$ the corresponding interpreted algebraic system. Given a theory family $T^{b} \in \operatorname{ThFam}(\mathcal{I})$, we set $\mathfrak{A}_{T^{b}}^{b}=$ $\left\langle\mathcal{A}^{b}, T^{b}\right\rangle$. 
Lemma 1 Let $\mathcal{I}=\left\langle\boldsymbol{A}^{b}, C\right\rangle$ be a r-institution and $T^{b} \in \operatorname{ThFam}(\mathcal{I})$. Then:

(1) $\mathfrak{A}_{T^{b}}^{b} \in \operatorname{MatSys}(\mathcal{I})$;

(2) For all $\Sigma \in\left|\mathbf{S i g n}^{b}\right|$ and $\Phi \cup\{\varphi\} \subseteq \operatorname{SEN}^{b}(\Sigma)$, if $\Phi \subseteq T_{\Sigma}^{b}$ and $\varphi \in C_{\Sigma}^{\mathfrak{A}^{b}} T^{b}(\Phi)$, then $\varphi \in T_{\Sigma}^{b}$.

\section{Proof.}

(1) Let $\Sigma \in\left|\mathbf{S i g n}^{b}\right|$ and $\Phi \cup\{\varphi\} \subseteq \operatorname{SEN}^{b}(\Sigma)$, such that $\varphi \in C_{\Sigma}(\Phi)$. By structurality, for all $\Sigma^{\prime} \in\left|\mathbf{S i g n}^{b}\right|$ and $f \in \operatorname{Sign}^{b}\left(\Sigma, \Sigma^{\prime}\right), \operatorname{SEN}^{b}(f)(\varphi) \epsilon$ $C_{\Sigma^{\prime}}\left(\operatorname{SEN}^{b}(f)(\Phi)\right)$. Thus, since $T^{b} \in \operatorname{ThFam}(\mathcal{I})$, if $\operatorname{SEN}^{b}(f)(\Phi) \subseteq T_{\Sigma^{\prime}}^{b}$, then $\operatorname{SEN}^{b}(f)(\varphi) \in T_{\Sigma^{\prime}}^{b}$. This proves that $\varphi \in C_{\Sigma}^{\mathfrak{A d}^{b}}(\Phi)$. Therefore $\mathfrak{A}_{T^{b}}^{b} \in \operatorname{MatSys}(\mathcal{I})$.

(2) This follows directly by the definition of $C^{\mathfrak{A}^{b}} T^{\mathfrak{b}}$.

The following is a version of the completeness theorem for sentential logics lifted to the level of $\pi$-institutions:

Proposition 2 Let $\boldsymbol{A}^{b}=\left\langle\mathbf{S i g n}^{b}, \mathrm{SEN}^{b}, N^{b}\right\rangle$ be a base algebraic system and $\mathcal{I}=\left\langle\boldsymbol{A}^{b}, C\right\rangle$ a $\pi$-institution based on $\boldsymbol{A}^{\mathrm{b}}$. Then, $C=C^{\operatorname{MatSys}(\mathcal{I})}$.

Proof. By definition, for all $\mathfrak{A} \in \operatorname{MatSys}(\mathcal{I})$, we have $C \leq C^{\mathfrak{A}}$. Therefore, $C \leq \bigcap\left\{C^{\mathfrak{A}}: \mathfrak{A} \in \operatorname{Mat} \operatorname{Sys}(\mathcal{I})\right\}=C^{\operatorname{MatSys}(\mathcal{I})}$.

Suppose, conversely, that $\Sigma \in\left|\mathbf{S i g n}^{b}\right|, \Phi \cup\{\varphi\} \subseteq \operatorname{SEN}^{b}(\Sigma)$, such that $\varphi \in C_{\Sigma}^{\operatorname{MatSys}(\mathcal{I})}(\Phi)$. To see that $\varphi \in C_{\Sigma}(\Phi)$, it must be shown that, for all $T^{b} \in \operatorname{ThFam}(\mathcal{I}), \Phi \subseteq T_{\Sigma}^{b}$ implies $\varphi \in T_{\Sigma}^{b}$. To see this, suppose that $T^{b} \in \operatorname{ThFam}(\mathcal{I})$, such that $\Phi \subseteq T_{\Sigma}^{b}$. Consider the pair $\mathfrak{A}_{T^{b}}^{b}=\left\langle\mathcal{A}^{b}, T^{b}\right\rangle$. By Part (1) of Lemma 1, $\mathfrak{A}_{T}^{b} \in \operatorname{MatSys}(\mathcal{I})$. Thus, by the hypothesis, $\varphi \in$ $C_{\Sigma}^{\mathfrak{A}^{\mathfrak{b}}}(\Phi)$. Since $\Phi \subseteq T_{\Sigma}^{b}$, by Part (2) of Lemma 1, $\varphi \in T_{\Sigma}^{b}$. This concludes the proof.

We denote by $\operatorname{MatSys}^{r}(\mathcal{I})$ the class of all $\mathcal{I}$-matrix systems $\mathfrak{A}=\langle\mathcal{A}, T\rangle$, with $\mathcal{A}=\langle\boldsymbol{A},\langle F, \alpha\rangle\rangle, \boldsymbol{A}=\langle$ Sign, SEN,$N\rangle$, such that, there exists $\Sigma \in|\mathbf{S i g n}|$, with $\left|T_{\Sigma}\right|=1$. We call members of $\operatorname{MatSys}^{r}(\mathcal{I})$ weak Rasiowa $\mathcal{I}$-matrix systems. 
We denote by $\operatorname{MatSys}^{R}(\mathcal{I})$ the class of all $\mathcal{I}$-matrix systems $\mathfrak{A}=\langle\mathcal{A}, T\rangle$, with $\mathcal{A}=\langle\boldsymbol{A},\langle F, \alpha\rangle\rangle, \boldsymbol{A}=\langle\operatorname{Sign}, \mathrm{SEN}, N\rangle$, such that, for all $\Sigma \in|\operatorname{Sign}|$, $\left|T_{\Sigma}\right|=1$. We call members of $\operatorname{MatSys}^{R}(\mathcal{I})$ strong Rasiowa $\mathcal{I}$-matrix systems.

In analogy with the sentential logic framework, we say that the weak (respectively, strong) Rasiowa semantics property holds for a $\pi$-institution $\mathcal{I}=\left\langle\boldsymbol{A}^{\mathrm{b}}, C\right\rangle$ if its closure system $C$ on $\boldsymbol{A}^{\mathrm{b}}$ is uniquely determined by the class of all weak (respectively, strong) Rasiowa $\mathcal{I}$-matrix systems.

\section{The Strong Wójcicki Condition}

Recall that, given a $\pi$-institution $\mathcal{I}=\left\langle\boldsymbol{A}^{\mathrm{b}}, C\right\rangle$ and a theory family $T^{\mathrm{b}} \epsilon$ $\operatorname{ThFam}(\mathcal{I})$, the Suszko congruence system $\widetilde{\Omega}^{\mathcal{I}}\left(T^{b}\right)$ of $T^{b}$ in $\mathcal{I}$ is the largest congruence system compatible with all theory families $T^{\prime b} \geq T^{b}$ of $\mathcal{I}$ (see Section 6 of [8]). In terms of the Leibniz congruence systems of the theories of $\mathcal{I}$ (Section 2 of [8]), we have

$$
\widetilde{\Omega}^{\mathcal{I}}\left(T^{b}\right)=\bigcap\left\{\Omega\left(T^{\prime b}\right): T^{b} \leq T^{\prime b} \in \operatorname{ThFam}(\mathcal{I})\right\} .
$$

A well-known characterization of the Suszko congruence system of a theory family $T^{b} \in \operatorname{ThFam}(\mathcal{I})$ (see, also, Section 6 of [8]) asserts that, for all $\Sigma \in\left|\operatorname{Sign}^{b}\right|$ and all $\varphi, \psi \in \operatorname{SEN}^{b}(\Sigma),\langle\varphi, \psi\rangle \in \widetilde{\Omega}_{\Sigma}^{\mathcal{I}}\left(T^{b}\right)$ if and only if, for all $\sigma: \operatorname{SEN}^{b+1} \rightarrow \operatorname{SEN}^{b}$ in $N^{b}, \Sigma^{\prime} \in\left|\mathbf{S i g n}^{b}\right|, f \in \operatorname{Sign}^{b}\left(\Sigma, \Sigma^{\prime}\right), \vec{\chi} \in \operatorname{SEN}^{b}\left(\Sigma^{\prime}\right)^{k}$,

$$
C_{\Sigma^{\prime}}\left(T_{\Sigma^{\prime}}^{b} \cup\left\{\sigma_{\Sigma^{\prime}}\left(\operatorname{SEN}^{b}(f)(\varphi), \vec{\chi}\right)\right\}\right)=C_{\Sigma^{\prime}}\left(T_{\Sigma^{\prime}}^{b} \cup\left\{\sigma_{\Sigma^{\prime}}\left(\operatorname{SEN}^{b}(f)(\psi), \vec{\chi}\right)\right\}\right),
$$

where Equation (2) is a shorthand for the equation in which, in $\sigma_{\Sigma^{\prime}}$ : $\operatorname{SEN}^{b}\left(\Sigma^{\prime}\right)^{k+1} \rightarrow \operatorname{SEN}^{b}\left(\Sigma^{\prime}\right)$, the $\Sigma^{\prime}$-sentences $\operatorname{SEN}^{b}(f)(\varphi), \operatorname{SEN}^{b}(f)(\psi)$ may occupy any position (not just the first) as long as they occupy the same position on the left and right hand sides.

A $\pi$-institution $\mathcal{I}=\left\langle\boldsymbol{A}^{b}, C\right\rangle$, with $\boldsymbol{A}^{\mathrm{b}}=\left\langle\mathbf{S i g n}^{\mathrm{b}}, \mathrm{SEN}^{\mathrm{b}}, N^{\mathrm{b}}\right\rangle$, satisfies the strong Wójcicki condition if, for all $T^{b} \in \operatorname{ThFam}(\mathcal{I})$, and all $\Sigma \in\left|\mathbf{S i g n}^{b}\right|$, $\varphi \in \operatorname{SEN}^{b}(\Sigma)$,

$$
\varphi \in T_{\Sigma}^{b} \quad \text { implies } T_{\Sigma}^{b}=\left\{\psi \in \operatorname{SEN}^{b}(\Sigma):\langle\varphi, \psi\rangle \in \widetilde{\Omega}_{\Sigma}^{\mathcal{I}}\left(T^{b}\right)\right\} .
$$

We now reformulate one half of Wójcicki's Conjecture and Malinowski's Theorem for logics formalized as $\pi$-institutions: 
Proposition 3 Let $\boldsymbol{A}^{b}=\left\langle\mathbf{S i g n}^{b}, \mathrm{SEN}^{b}, N^{b}\right\rangle$ be a base algebraic system and $\mathcal{I}=\left\langle\boldsymbol{A}^{b}, C\right\rangle$ a $\pi$-institution based on $\boldsymbol{A}^{b}$. If $\mathcal{I}$ satisfies the strong Wójcicki condition, then $\mathcal{I}$ has the weak Rasiowa semantics property.

Proof. Suppose that $\mathcal{I}=\left\langle A^{b}, C\right\rangle$ satisfies the strong Wójcicki condition. Let $\mathcal{A}^{b}=\left\langle\boldsymbol{A}^{b},\left\langle I_{\mathbf{S i g n}^{b}}, \iota^{b}\right\rangle\right\rangle$, where $\left\langle I_{\mathbf{S i g n}^{b}}, \iota^{b}\right\rangle: \boldsymbol{A}^{b} \rightarrow \boldsymbol{A}^{b}$ is the identity morphism. Consider, for all $T^{b} \in \operatorname{ThFam}(\mathcal{I})-\{\varnothing\}$, where $\varnothing=\{\varnothing\}_{\Sigma \in\left|\mathbf{S i g n}^{b}\right|}$, the $\mathcal{I}$-matrix system

$$
\mathfrak{A}^{T^{b}}=\left\langle\mathcal{A}^{b}, T^{b}\right\rangle .
$$

Clearly, the collection $\left\{\mathfrak{A}^{T^{b}}: T^{b} \in \operatorname{ThFam}(\mathcal{I})-\{\varnothing\}\right\}$ is a strongly adequate matrix system semantics for $\mathcal{I}$. Now consider the collection

$$
\left\{\mathfrak{A}^{T^{b}} / \widetilde{\Omega}^{\mathcal{I}}\left(T^{b}\right)=\left\langle\mathcal{A}^{b} / \widetilde{\Omega}^{\mathcal{I}}\left(T^{b}\right), T^{b} / \widetilde{\Omega}^{\mathcal{I}}\left(T^{b}\right)\right\rangle: T^{b} \in \operatorname{ThFam}(\mathcal{I})-\{\varnothing\}\right\} .
$$

The latter is, by the strong Wójcicki condition, a collection of weak Rasiowa $\mathcal{I}$-matrix systems that is strongly adequate for $\mathcal{I}$. Therefore, $\mathcal{I}$ has the weak Rasiowa semantics property.

Note that to carry out the construction in the proof of Proposition 3 one has to divide by the Suszko congruence systems. Invariance under signature morphisms is necessary for this quotient construction to make sense.

\section{The Weak Wójcicki Condition}

Given a $\pi$-institution $\mathcal{I}=\left\langle\boldsymbol{A}^{b}, C\right\rangle$ and a theory family $T^{b} \in \operatorname{ThFam}(\mathcal{I})$, the Suszko congruence family $\widetilde{\Theta}^{\mathcal{I}}\left(T^{b}\right)$ of $T^{b}$ in $\mathcal{I}$ is the largest congruence family compatible with all theory families $T^{\prime b} \geq T^{b}$ of $\mathcal{I}$. Contrast this with the Suszko congruence system $\widetilde{\Omega}^{\mathcal{I}}\left(T^{b}\right)$, which is, of course, required to be a system, i.e., invariant under signature morphisms. A characterization of the Suszko congruence family of $T^{b} \in \operatorname{ThFam}(\mathcal{I})$, analogous to that of the Suszko congruence system, follows along the lines of the characterization of the Suszko congruence of a theory of a sentential logic, as given by Czelakowski in [3]. 
Proposition 4 Let $\mathcal{I}=\left\langle\boldsymbol{A}^{b}, C\right\rangle$, with $\boldsymbol{A}^{\mathrm{b}}=\left\langle\mathbf{S i g n}^{\mathrm{b}}, \mathrm{SEN}^{\mathrm{b}}, N^{\mathrm{b}}\right\rangle$, be a $\pi$ institution and $T^{b} \in \operatorname{ThFam}(\mathcal{I})$. For all $\Sigma \in\left|\operatorname{Sign}^{b}\right|$ and all $\varphi, \psi \in \operatorname{SEN}^{b}(\Sigma)$, $\langle\varphi, \psi\rangle \in \widetilde{\Theta}_{\Sigma}^{\mathcal{I}}\left(T^{b}\right)$ if and only if, for all $\sigma: \mathrm{SEN}^{b^{k+1}} \rightarrow \mathrm{SEN}^{b}$ in $N^{b}$ and all $\vec{\chi} \in \operatorname{SEN}^{b}(\Sigma)^{k}$,

$$
C_{\Sigma}\left(T_{\Sigma}^{b} \cup\left\{\sigma_{\Sigma}(\varphi, \vec{\chi})\right\}\right)=C_{\Sigma}\left(T_{\Sigma}^{b} \cup\left\{\sigma_{\Sigma}(\psi, \vec{\chi})\right\}\right) .
$$

Proof. Define $\theta=\left\{\theta_{\Sigma}\right\}_{\Sigma \epsilon\left|\mathbf{S i g n}^{\mathrm{b}}\right|}$, for all $\Sigma \in\left|\mathbf{S i g n}^{\mathrm{b}}\right|$ and all $\varphi, \psi \epsilon$ $\operatorname{SEN}^{b}(\Sigma)$, by setting

$$
\begin{aligned}
\langle\varphi, \psi\rangle \in \theta_{\Sigma} \quad \text { iff } & \text { for all } \sigma: \mathrm{SEN}^{\mathrm{k}+1} \rightarrow \operatorname{SEN}^{\mathrm{b}} \text { in } N^{\mathrm{b}}, \vec{\chi} \in \operatorname{SEN}^{\mathrm{b}}(\Sigma)^{k} \\
& C_{\Sigma}\left(T_{\Sigma}^{\mathrm{b}} \cup\left\{\sigma_{\Sigma}(\varphi, \vec{\chi})\right\}\right)=C_{\Sigma}\left(T_{\Sigma}^{\mathrm{b}} \cup\left\{\sigma_{\Sigma}(\psi, \vec{\chi})\right\}\right) .
\end{aligned}
$$

It is easy to see that $\theta$ is an equivalence family on $\operatorname{SEN}^{b}$. To see that it is a congruence of $\boldsymbol{A}^{\mathrm{b}}$, consider $\tau: \mathrm{SEN}^{b^{\ell}} \rightarrow \mathrm{SEN}^{\mathrm{b}}$ in $N^{\mathrm{b}}$ and $\vec{\varphi}, \vec{\psi} \in \operatorname{SEN}^{\mathrm{b}}(\Sigma)^{\ell}$, such that $\vec{\varphi} \theta_{\Sigma}^{\ell} \vec{\psi}$. Then, we have, for all $\sigma: \mathrm{SEN}^{\mathrm{b}^{k+1}} \rightarrow \mathrm{SEN}^{\mathrm{b}}$ in $N^{\mathrm{b}}$ and all $\vec{\chi} \in \operatorname{SEN}^{b}(\Sigma)^{k}$,

$$
\begin{aligned}
C_{\Sigma}\left(T_{\Sigma}^{b} \cup\left\{\sigma_{\Sigma}\left(\tau_{\Sigma}(\vec{\varphi}), \vec{\chi}\right)\right\}\right) & =C_{\Sigma}\left(T_{\Sigma}^{b} \cup\left\{\sigma_{\Sigma}\left(\tau_{\Sigma}\left(\psi_{0}, \varphi_{1}, \ldots, \varphi_{\ell-1}\right), \vec{\chi}\right)\right\}\right) \\
& =C_{\Sigma}\left(T_{\Sigma}^{b} \cup\left\{\sigma_{\Sigma}\left(\tau_{\Sigma}\left(\psi_{0}, \psi_{1}, \ldots, \varphi_{\ell-1}\right), \vec{\chi}\right)\right\}\right) \\
& =\cdots \\
& =C_{\Sigma}\left(T_{\Sigma}^{b} \cup\left\{\sigma_{\Sigma}\left(\tau_{\Sigma}(\vec{\psi}), \vec{\chi}\right)\right\}\right),
\end{aligned}
$$

whence $\tau_{\Sigma}(\vec{\varphi}) \theta_{\Sigma} \tau_{\Sigma}(\vec{\psi})$ and $\theta$ is a congruence family of $\boldsymbol{A}^{b}$. To see that $\theta$ is compatible with all theory families $T^{\prime b} \geq T^{b}$, assume $\langle\varphi, \psi\rangle \in \theta_{\Sigma}$ and $\varphi \in T_{\Sigma}^{\prime b}$. Then, we have

$$
\begin{array}{rlrl}
\psi & \in C_{\Sigma}\left(T_{\Sigma}^{b} \cup\{\psi\}\right) & \\
& =C_{\Sigma}\left(T_{\Sigma}^{b} \cup\{\varphi\}\right) & & \text { (since } \left.\langle\varphi, \psi\rangle \in \theta_{\Sigma}\right) \\
& \subseteq C_{\Sigma}\left(T_{\Sigma}^{\prime b} \cup\{\varphi\}\right) & & \text { (since } \left.T^{b} \leq T^{\prime b}\right) \\
& =C_{\Sigma}\left(T_{\Sigma}^{\prime b}\right) & & \left(\text { since } \varphi \in T_{\Sigma}^{\prime b}\right) \\
& =T_{\Sigma}^{\prime b} . & & \left(\text { since } T^{\prime b} \in \operatorname{ThFam}(\mathcal{I})\right)
\end{array}
$$

Finally, to see that $\theta$ is the largest congruence family compatible with $T^{b}$, suppose that $\eta$ is such a family and $\langle\varphi, \psi\rangle \in \eta_{\Sigma}$. Then, for all $\sigma$ : $\mathrm{SEN}^{b^{k+1}} \rightarrow \mathrm{SEN}^{\mathrm{b}}$ in $N^{\mathrm{b}}$ and all $\vec{\chi} \in \operatorname{SEN}^{\mathrm{b}}(\Sigma)^{k}$, by the congruence property of $\eta, \sigma_{\Sigma}(\varphi, \vec{\chi}) \eta_{\Sigma} \sigma_{\Sigma}(\psi, \vec{\chi})$. Therefore, by compatibility of $\eta$ with all theory families of $\mathcal{I}$ including $T^{b}$, we get that $C_{\Sigma}\left(T_{\Sigma}^{b} \cup\left\{\sigma_{\Sigma}(\varphi, \vec{\chi})\right\}\right)=C_{\Sigma}\left(T_{\Sigma}^{b} \cup\right.$ $\left.\left\{\sigma_{\Sigma}(\psi, \vec{\chi})\right\}\right)$. This shows that $\langle\varphi, \psi\rangle \in \theta_{\Sigma}$. Thus, $\eta \leq \theta$. 
Since $\theta$ is the largest congruence family of $\mathcal{I}$ that is compatible with $T^{b} \in \operatorname{ThFam}(\mathcal{I})$, we get, by definition, $\theta=\widetilde{\Theta}^{\mathcal{I}}\left(T^{b}\right)$.

Either directly from the fact that every congruence system is also a congruence family, or based on the corresponding characterizations, we obtain the following corollary:

Corollary 5 Let $\mathcal{I}=\left\langle\boldsymbol{A}^{\mathrm{b}}, C\right\rangle$, with $\boldsymbol{A}^{\mathrm{b}}=\left\langle\mathbf{S i g n}^{\mathrm{b}}, \mathrm{SEN}^{\mathrm{b}}, N^{\mathrm{b}}\right\rangle$, be a $\pi$ institution. For all $T^{\mathrm{b}} \in \operatorname{ThFam}(\mathcal{I})$,

$$
\widetilde{\Omega}^{\mathcal{I}}\left(T^{b}\right) \leq \widetilde{\Theta}^{\mathcal{I}}\left(T^{b}\right) .
$$

A $\pi$-institution $\mathcal{I}=\left\langle\boldsymbol{A}^{\mathrm{b}}, C\right\rangle$ based on $\boldsymbol{A}^{\mathrm{b}}=\left\langle\mathbf{S i g n}^{\mathrm{b}}, \mathrm{SEN}^{\mathrm{b}}, N^{\mathrm{b}}\right\rangle$ satisfies the weak Wójcicki condition if, for all $T^{b} \in \operatorname{ThFam}(\mathcal{I})$ and all $\Sigma \in\left|\mathbf{S i g n}^{b}\right|$, $\varphi \in \operatorname{SEN}^{b}(\Sigma)$,

$$
\varphi \in T_{\Sigma}^{b} \quad \text { implies } T_{\Sigma}^{b}=\left\{\psi \in \operatorname{SEN}^{b}(\Sigma):\langle\varphi, \psi\rangle \in \widetilde{\Theta}_{\Sigma}^{\mathcal{I}}\left(T^{b}\right)\right\} .
$$

We are now ready to formulate the second half of Wójcicki's Conjecture and Malinowski's Theorem for logics formalized as $\pi$-institutions:

Proposition 6 Let $\boldsymbol{A}^{b}=\left\langle\mathbf{S i g n}^{b}, \mathrm{SEN}^{b}, N^{b}\right\rangle$ be an algebraic system and $\mathcal{I}=\left\langle\boldsymbol{A}^{b}, C\right\rangle$ a $\pi$-institution based on $\boldsymbol{A}^{\mathrm{b}}$. If $\mathcal{I}$ has the strong Rasiowa semantics property, then it satisfies the weak Wójcicki condition.

Proof. Suppose that $\mathcal{I}=\left\langle\boldsymbol{A}^{b}, C\right\rangle$ has the strong Rasiowa semantics property. Let $T^{b} \in \operatorname{ThFam}(\mathcal{I}), \Sigma \in\left|\operatorname{Sign}^{b}\right|, \varphi \in \operatorname{SEN}^{b}(\Sigma)$, such that $\varphi \in T_{\Sigma}^{b}$. Set

$$
[\varphi]=\left\{\psi \in \operatorname{SEN}^{b}(\Sigma):\langle\varphi, \psi\rangle \in \widetilde{\Theta}_{\Sigma}^{\mathcal{I}}\left(T^{b}\right)\right\} .
$$

We must show that $T_{\Sigma}^{b}=[\varphi]$. We follow the proof of the corresponding result in [5].

$\subseteq$ : Assume that there exists $\psi \in T_{\Sigma}^{b}$, such that $\psi \notin[\varphi]$. Since $\langle\varphi, \psi\rangle \notin$ $\widetilde{\Theta}_{\Sigma}^{\mathcal{I}}\left(T^{b}\right)$, by Proposition 4, there exists $\sigma: \mathrm{SEN}^{\mathrm{b}^{k+1}} \rightarrow \mathrm{SEN}^{\mathrm{b}}$ in $N^{\mathrm{b}}$ and $\vec{\chi} \in \operatorname{SEN}^{b}(\Sigma)^{k}$, such that

$$
C_{\Sigma}\left(T_{\Sigma}^{b} \cup\left\{\sigma_{\Sigma}(\varphi, \vec{\chi})\right\}\right) \neq C_{\Sigma}\left(T_{\Sigma}^{b} \cup\left\{\sigma_{\Sigma}(\psi, \vec{\chi})\right\}\right) .
$$

Assume, without loss of generality due to symmetry, that

$$
\xi \in C_{\Sigma}\left(T_{\Sigma}^{b} \cup\left\{\sigma_{\Sigma}(\varphi, \vec{\chi})\right\}\right) \text { and } \xi \notin C_{\Sigma}\left(T_{\Sigma}^{b} \cup\left\{\sigma_{\Sigma}(\psi, \vec{\chi})\right\}\right) .
$$


By the strong Rasiowa semantics property, there exists a strong Rasiowa $\mathcal{I}$-matrix system $\mathfrak{A}=\langle\langle\boldsymbol{A},\langle F, \alpha\rangle\rangle, T\rangle, \Sigma^{\prime} \in\left|\mathbf{S i g n}^{\mathrm{b}}\right|$ and $f \in$ $\operatorname{Sign}^{b}\left(\Sigma, \Sigma^{\prime}\right)$, such that

$$
\alpha_{\Sigma^{\prime}}\left(\operatorname{SEN}^{b}(f)\left(T_{\Sigma}^{b}\right)\right) \cup\left\{\alpha_{\Sigma^{\prime}}\left(\operatorname{SEN}^{b}(f)\left(\sigma_{\Sigma}(\psi, \vec{\chi})\right)\right)\right\} \subseteq T_{F\left(\Sigma^{\prime}\right)}
$$

whereas

$$
\alpha_{\Sigma^{\prime}}\left(\operatorname{SEN}^{b}(f)(\xi)\right) \notin T_{F\left(\Sigma^{\prime}\right)} .
$$

On the other hand, notice that, since $\mathfrak{A}$ is an $\mathcal{I}$-matrix system and $\xi \in C_{\Sigma}\left(T_{\Sigma}^{b} \cup\left\{\sigma_{\Sigma}(\varphi, \vec{\chi})\right\}\right)$, we must have

$$
\alpha_{\Sigma^{\prime}}\left(\operatorname{SEN}^{b}(f)\left(\sigma_{\Sigma}(\varphi, \vec{\chi})\right)\right) \notin T_{F\left(\Sigma^{\prime}\right)} .
$$

Finally, the fact that $\varphi, \psi \in T_{\Sigma}^{b}$ and $\alpha_{\Sigma^{\prime}}\left(\operatorname{SEN}^{b}(f)\left(T_{\Sigma}^{b}\right)\right) \subseteq T_{F\left(\Sigma^{\prime}\right)}$ ensures that $\alpha_{\Sigma^{\prime}}\left(\operatorname{SEN}^{b}(f)(\varphi)\right) \in T_{F\left(\Sigma^{\prime}\right)}$ and $\alpha_{\Sigma^{\prime}}\left(\operatorname{SEN}^{b}(f)(\psi)\right) \in T_{F\left(\Sigma^{\prime}\right)}$. Taking into account the commutativity of the following diagram,

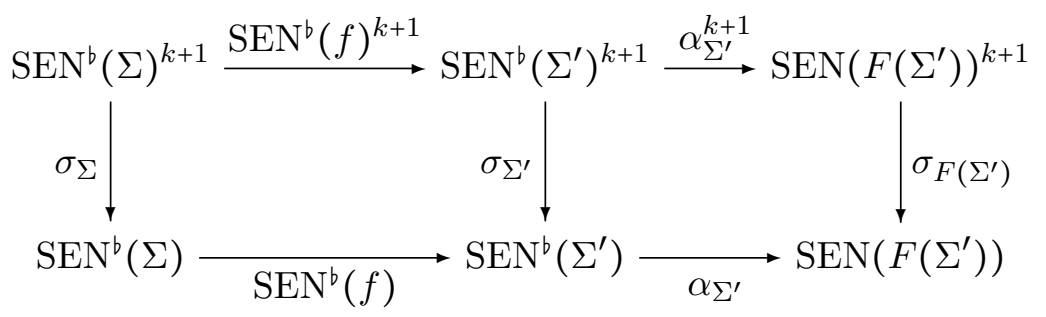

we have obtained the following four relations:

$$
\begin{array}{rll}
\alpha_{\Sigma^{\prime}}\left(\operatorname{SEN}^{b}(f)(\varphi)\right) & \in T_{F\left(\Sigma^{\prime}\right)} \\
\alpha_{\Sigma^{\prime}}\left(\operatorname{SEN}^{b}(f)(\psi)\right) & \in T_{F\left(\Sigma^{\prime}\right)} \\
\sigma_{F\left(\Sigma^{\prime}\right)}\left(\alpha_{\Sigma^{\prime}}\left(\operatorname{SEN}^{b}(f)(\varphi)\right), \alpha_{\Sigma^{\prime}}^{k}\left(\operatorname{SEN}^{b}(f)^{k}(\vec{\chi})\right)\right) & \notin & T_{F\left(\Sigma^{\prime}\right)} \\
\sigma_{F\left(\Sigma^{\prime}\right)}\left(\alpha_{\Sigma^{\prime}}\left(\operatorname{SEN}^{b}(f)(\psi)\right), \alpha_{\Sigma^{\prime}}^{k}\left(\operatorname{SEN}^{b}(f)^{k}(\vec{\chi})\right)\right) & \epsilon & T_{F\left(\Sigma^{\prime}\right)} .
\end{array}
$$

These are clearly contradictory in view of the hypothesis that $\mathfrak{A}$ is a strong Rasiowa $\mathcal{I}$-matrix system, i.e., $\left|T_{F\left(\Sigma^{\prime}\right)}\right|=1$.

卫: Suppose, next, that $\psi \in[\varphi]$, i.e., that $\langle\varphi, \psi\rangle \in \widetilde{\Theta}_{\Sigma}^{\mathcal{I}}\left(T^{b}\right)$. Then, we have

$$
\begin{array}{rlrl}
\psi & \in C_{\Sigma}\left(T_{\Sigma}^{b} \cup\{\psi\}\right) & \\
& =C_{\Sigma}\left(T_{\Sigma}^{b} \cup\{\varphi\}\right) & & \left(\text { since }\langle\varphi, \psi\rangle \in \widetilde{\Theta}_{\Sigma}^{\mathcal{I}}\left(T^{b}\right)\right) \\
& =C_{\Sigma}\left(T_{\Sigma}^{b}\right) & & \left(\text { since } \varphi \in T_{\Sigma}^{b}\right)
\end{array}
$$

We now conclude that, if $\mathcal{I}$ has the strong Rasiowa semantics property, then it satisfies the weak Wójcicki condition. 


\section{Conclusion}

We have established the following implications among conditions related to the Rasiowa semantics property and the Wójcicki condition applicable for logics formalized as $\pi$-institutions:

Theorem 7 Let $\boldsymbol{A}^{b}=\left\langle\mathbf{S i g n}^{b}, \mathrm{SEN}^{b}, N^{b}\right\rangle$ be an algebraic system and $\mathcal{I}=\left\langle\boldsymbol{A}^{\mathrm{b}}, C\right\rangle$ a $\pi$-institution based on $\boldsymbol{A}^{\mathrm{b}}$.

(1) If $\mathcal{I}$ satisfies the strong Wójcicki condition, then it has the weak Rasiowa semantics property.

(2) If $\mathcal{I}$ has the strong Rasiowa semantics property, then it satisfies the weak Wójcicki condition.

Pictorially, we have

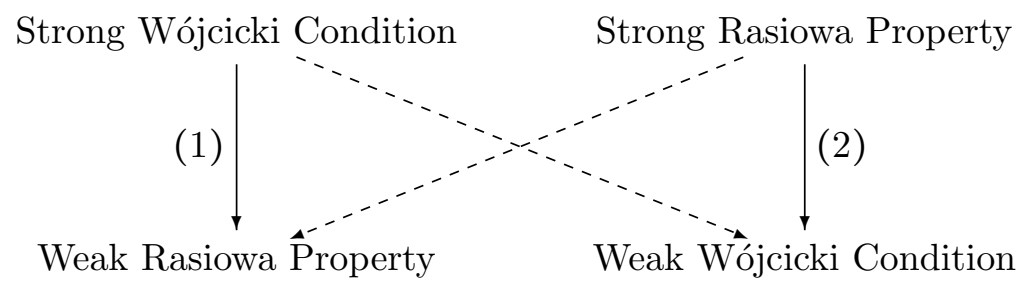

where the dashed implications are trivial.

Proof. (1) is Proposition 3 and (2) is Proposition 6.

Note that, if $\boldsymbol{A}^{\mathrm{b}}$ is an algebraic system with the trivial signature category, all congruence families of $\boldsymbol{A}^{\mathrm{b}}$ are also congruence systems. In particular, for a $\pi$-institution based on such an algebraic system, the Suszko congruence system $\widetilde{\Omega}^{\mathcal{I}}\left(T^{b}\right)$ and the Suszko congruence family $\widetilde{\Theta}^{\mathcal{I}}\left(T^{b}\right)$ of a theory family $T^{b} \in \operatorname{ThFam}(\mathcal{I})$ coincide. Thus, $\mathcal{I}$ satisfies the strong Wójcicki condition if and only if it satisfies the weak Wójcicki condition. Moreover, in this case, the strong and the weak Rasiowa semantics properties coincide. Therefore, we obtain the following corollary, an analog for logics formalized as $\pi$-institutions of the Wójcicki-Malinowski Theorem that motivated our work:

Corollary 8 Let $\boldsymbol{A}^{b}=\left\langle\operatorname{Sign}^{b}, \mathrm{SEN}^{b}, N^{b}\right\rangle$ be an algebraic system, with the trivial signature category, and $\mathcal{I}=\left\langle\boldsymbol{A}^{b}, C\right\rangle$ a $\pi$-institution based on $\boldsymbol{A}^{b}$. $\mathcal{I}$ has the Rasiowa semantics property if and only if it satisfies the Wójcicki condition. 


\section{References}

[1] W.J. Blok and D. Pigozzi, Algebraizable Logics, Memoirs of the American Mathematical Society, Vol. 77, No. 396 (1989).

[2] J. Czelakowski, Reduced Products of Logical Matrices, Studia Logica 39 (1980), 19-43.

[3] J. Czelakowski, The Suszko Operator Part I, Studia Logica 74:1-2 (2003), 181-231.

[4] J.M. Font and R. Jansana, A General Algebraic Semantics for Sentential Logics, Lecture Notes in Logic, Vol. 332, No. 7 (1996), Springer-Verlag, Berlin Heidelberg, 1996.

[5] G. Malinowski, A Proof of Ryszard Wójcicki's Conjecture, Bulletin of the Section of Logic 7:1 (1978), 20-25.

[6] H. Rasiowa, An Algebraic Approach to Non-Classical Logics, Studies in Logic and the Foundations of Mathematics, Elsevier Science, 1974.

[7] R. Wójcicki, Matrix Approach in Methodology of Sentential Calculi, Studia Logica 32:1 (1973), 7-37.

[8] G. Voutsadakis, Categorical Abstract Algebraic Logic: Prealgebraicity and Protoalgebraicity, Studia Logica 85:2 (2007), 215-249.

[9] G. Voutsadakis, Categorical Abstract Algebraic Logic: The Subdirect Product Theorem, available in http://www. voutsadakis.com/RESEARCH/papers.html

School of Mathematics and Computer Science

Lake Superior State University

Sault Sainte Marie, MI 49783, USA

gvoutsad@lssu.edu 